\title{
A small shipborne desalination plant based on the principle of loop heat pipe
}

\author{
Tongyao Miao ${ }^{1,}{ }^{*}$, Zheng Fang ${ }^{1}$, Lingzhi Feng ${ }^{2}$, Yunhui Peng ${ }^{2}$ \\ ${ }^{1}$ School of Navigation, Wuhan University Of Technology, Wuhan, Hubei Province, 430061, China \\ ${ }^{2}$ School of Materials Science and Engineering, Wuhan University Of Technology, Wuhan, Hubei Province, 430070, China
}

\begin{abstract}
As is well-known, the problem of fresh water has always been a major problem for ocean navigation. The existing marine seawater desalination equipment generally has the disadvantages of large volume, high energy consumption and easy to pollute the environment. Based on this, we proposed a small seawater desalination system based on phase change to enhance heat transfer, which realized low temperature and low energy consumption by using the loop heat pipe technology, a new type of heat sink type spoiler evaporator and an integrated equal-heat plate fin condenser. The device is suitable for islands, fishing boats and other complicated areas where power is scarce and fresh water resources are scarce. It can also be used as fresh water emergency equipment for large ships, saving energy and being portable.
\end{abstract}

\section{System design}

This work main body keep evaporation system, cooling system, vacuum system, circulating spray system as well as fresh water collection system together, and also design the supporting base and limit device, structure as shown in fig. 1-1.

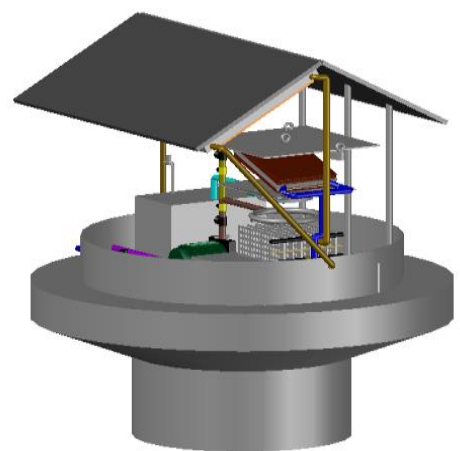

Fig. 1-1 Overall structure

\subsection{Evaporation system}

The evaporating system is composed of plate-type loop heat pipe, solar energy collecting plate, electric heating plate and radiator baffle evaporator. The specific structure is shown in figure 1-2.

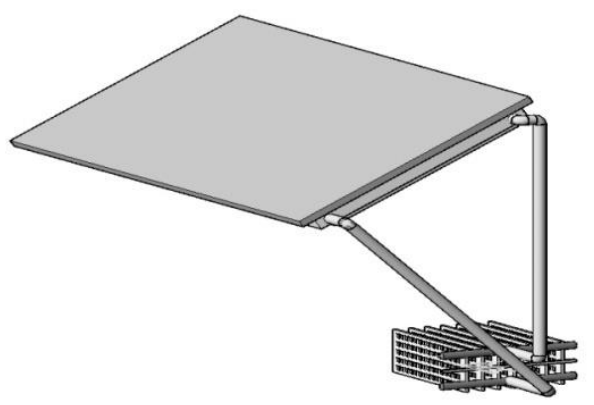

Fig. 1-2 Structure of evaporation system

The solar energy collection board and the electric heating board constitute the plate-type loop heat pipe on both sides of the system to collect heat distribution, and the backlit side is set on the electric heating board, and the heat source is mainly solar energy to collect heat, and the electric heating board provides electrical auxiliary heating when necessary. The heat transfer medium phase in the loop heat pipe transforms the heat to the radiator baffle evaporator, which is placed inside the tank to evaporate seawater.

Working principle of the loop heat pipe as shown in figure 1-3, evaporator absorbs heat at work, makes the capillary core inside the liquid medium evaporate, evaporation of fine holes on the gas by capillary core spreading, capillary core by evaporation circuit to condensing side, the steam in the cooling side cooling liquid, liquid medium by the capillary core after capillary force back into the capillary core inside, complete a cycle, achieve heat transfer. Compared with the traditional single-phase heat transfer, the two-phase heat transfer can improve the heat transfer efficiency and reduce the heat loss in the heat transfer process. 


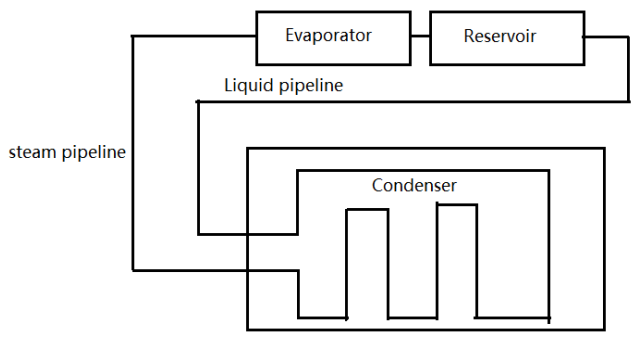

Fig. 1-3 Loop heat pipe working principle

\subsection{Condensation system}

The main body of the condensing system is composed of cold water inlet, cold water outlet and integrated isothermal plate-fin condenser. The flow of cold water inlet and cold water outlet is controlled by the throttle valve, as shown in figure 1-4.

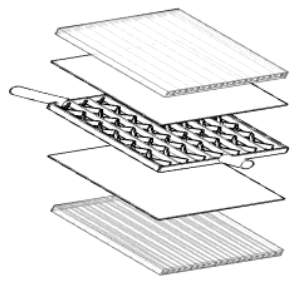

Fig. 1-4 The structure of condensation system

In principle, the uniform plate is similar to the heat pipe, but the conduction mode is different. The heat pipe is a linear heat conduction, while the heat in the uniform temperature plate is conducted on a two-dimensional plane, so it is more efficient and energy saving.

\subsection{Vacuum maintenance system}

The device adopts a sealed structure to maintain the internal pressure of the device and uses venturi principle to vacuum the device so that the internal pressure of the device can be reduced to reduce the boiling point of seawater evaporation.

\section{4. freshwater collection system}

The fresh water collection system consists of an inner and outer ring water plate, a fresh water collection barrel and a fresh water pipeline. The tank body is connected with the fresh water collecting barrel through the fresh water pipeline to ensure that the internal pressure of both is consistent and the device works more stably. As shown in figure 1-5, mist spraying seawater in plate fin disturbed flow evaporator heat evaporation, the steam rises, and the continuous integration of cold water soaking contact plate fin condenser, steam cooled liquid, condenses into droplets, under the action of gravity drop to the inside and outside the ring water pans, through fresh water pipe flow to the fresh water collection barrel, realize water collection.

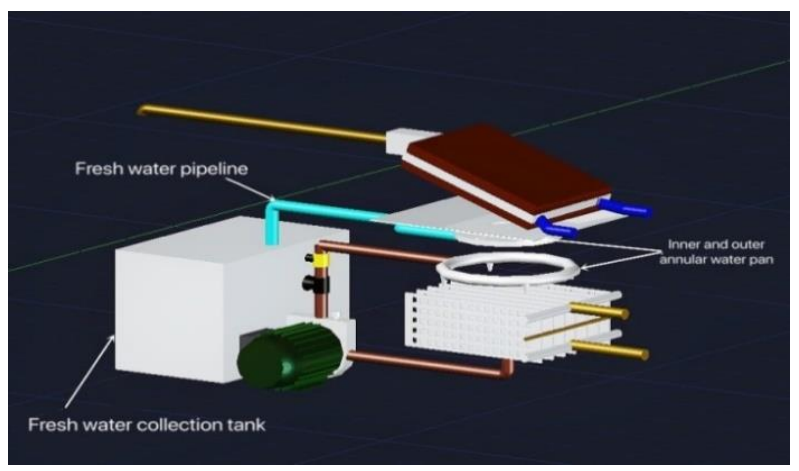

Fig. 1-5 Structure of freshwater collection system

\subsection{Circulating spray system}

The seawater circulating spray system is set in the vacuum system. The seawater circulating spray system includes the circulating spray pump, the atomizing spray head and the circulation pipeline. The atomizing spray head is set at the upper part of the condensing end; by setting up the circulating spray heat exchange system, the evaporator heat absorbed in the unevaporated seawater can be utilized to maximize the conversion of seawater into fresh water.

\section{6. base design and limit device}

Base is mainly composed of wave guardrail and the overall circuit parts, structure is shown in figure 1-6, the lower the overall structure is composed at the bottom of the cylinder and the inverted frustum of a cone, cone at the bottom of the cylinder with a transparent organic glass baffle rigid connection, in does not affect the premise of solar energy, avoid the interference of wind and waves, on the other hand, in the cylindrical baffle and the location of the frustum of a cone connected eight hole design, will the water export platform at the bottom of the platform, the cylinder diameter of $1 \mathrm{~m}, 1.5 \mathrm{~m}$ high, put solar panels and batteries inside circuit board.

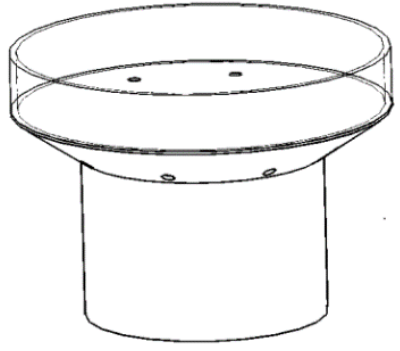

Fig. 1-6 Structure of the base

The structure of the limit device is shown in figure 1-7. The limit device is a rectangular block with a groove in the middle, which is located on the lower surface of the platform base. The groove is connected with the anchor chain. After calculation, the cuboid is $20 \mathrm{~cm}$ long, $10 \mathrm{~cm}$ wide and $7 \mathrm{~cm}$ high. The groove is $20 \mathrm{~cm}$ long, $2 \mathrm{~cm}$ wide and $5 \mathrm{~cm}$ high. Among them, the size of the groove matches the size of the anchor chain link, so that part of the anchor chain near the platform can only rotate in the space between the two walls of the groove relative to the 
platform. In addition, the longitudinal direction of the groove is parallel to the straight line determined by the direction of the center of gravity of the main body of the device.

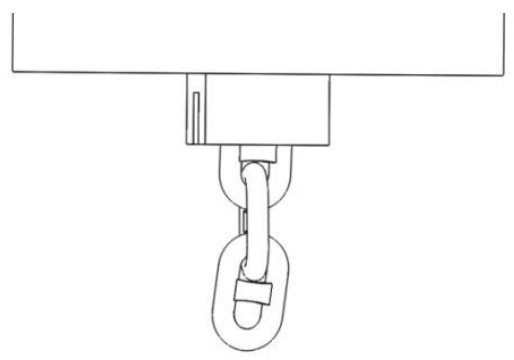

Fig. 1-7 Structure of the limit divice

\section{Fresh water production rate}

According to the independent function of each system and the function of the whole system, the mathematical analysis based on the dynamic model is established, and the analytical solution is obtained.

Table 2-1 The symbols and meanings of the substances

\begin{tabular}{cc}
\hline $\begin{array}{c}\text { Variable } \\
\text { symbol }\end{array}$ & Meaning \\
\hline$[\mathrm{A}]$ & $\begin{array}{c}\text { The evaporative liquid involved in the } \\
\text { reaction in the device } \\
\text { Water vapor that evaporates from the } \\
\text { seawater involved in the reaction }\end{array}$ \\
{$[\mathrm{B}]$} & $\begin{array}{c}\text { Fresh water produced by condensation } \\
\text { Gas not involved in the reaction in the } \\
\text { device }\end{array}$ \\
{$\left[\mathrm{B}^{\prime}\right]$} & $\begin{array}{c}\text { The proportion of water vapor in all gases } \\
\text { The proportion of other gases in all the } \\
\text { gases }\end{array}$ \\
{$\left[\mathrm{y}_{\mathrm{B}}\right]$} & $\begin{array}{c}\text { The proportion of other solutes in the } \\
\text { evaporation solution }\end{array}$ \\
$\mathrm{x}$ &
\end{tabular}

Seawater keeps coming in, and the amount stays the same. The series system constraint of the first-order reaction is:

$$
\begin{gathered}
\frac{\mathrm{d}[\mathrm{A}]}{\mathrm{dt}}=\mathrm{e} \\
\frac{\mathrm{d}[\mathrm{B}]}{\mathrm{dt}}=\mathrm{k}_{1}[\mathrm{~A}]-\mathrm{k}_{2}[\mathrm{~B}] \\
\frac{\mathrm{d}[\mathrm{C}]}{\mathrm{dt}}=\mathrm{k}_{2}[\mathrm{~B}]+\mathrm{l}(t)
\end{gathered}
$$

The amount of sea water remains unchanged at $[\mathrm{A}] 0$, that is $[\mathrm{A}] \sim \mathrm{N}([\mathrm{A}] 0$, ):

$$
[B]=-\frac{k_{1}[A]_{0}}{k_{2}} e^{-k_{2} t}+\frac{k_{1}[A]_{0}}{k_{2}}
$$

The resulting differential equation for desalinated water is,

$$
\frac{\mathrm{d}[\mathrm{C}]}{\mathrm{dt}}=-\mathrm{k}_{1}[\mathrm{~A}]_{0} \mathrm{e}^{-\mathrm{k}_{2} \mathrm{t}}+\mathrm{k}_{1}[\mathrm{~A}]_{0}
$$

The time-varying function of the desalinated water is,

$$
[C]=\frac{\mathrm{k}_{1}[\mathrm{~A}]_{0}}{\mathrm{k}_{2}} \mathrm{e}^{-\mathrm{k}_{2} \mathrm{t}}+\mathrm{k}_{1}[\mathrm{~A}]_{0} \mathrm{t}-\frac{\mathrm{k}_{1}[\mathrm{~A}]_{0}}{\mathrm{k}_{2}}
$$

According to the properties of the analytic solution function, it can be seen that the total amount of sea water in the device remains stable, with the passage of time, water vapor tends to be stable at $\mathrm{k}_{1}[\mathrm{~A}]_{0} / \mathrm{k}_{2}$, and fresh water tends to be stable at $\mathrm{k}_{1}[\mathrm{~A}]_{0}$. The rate at which water molecules leave the surface should be the same, so you can calculate the rate at which water molecules in

\begin{tabular}{|c|c|c|}
\hline $\begin{array}{l}\text { Constant } \\
\text { symbol }\end{array}$ & Numerical value & Meaning \\
\hline$m$ & $\begin{array}{l}\text { Mass of a single water } \\
\text { molecule }\end{array}$ & $3 \times 10^{\wedge}(-26) \mathrm{kg}$ \\
\hline$k_{B}$ & Boltzmann constant & 1. $38 \times 10^{\wedge}(-23) \mathrm{J} / \mathrm{K}$ \\
\hline$N_{A}$ & Avogadro constant & $6.02 \times 10^{\wedge} 23$ \\
\hline$R$ & Ideal gas constant & $8.314 \mathrm{~J} \cdot \mathrm{mol}^{-1} \cdot \mathrm{K}^{-1}$ \\
\hline $\mathrm{S}^{*}{ }_{1}$ & $\begin{array}{l}\text { Relative heat transfer area } \\
\text { of evaporation section }\end{array}$ & 0.327 \\
\hline $\mathbf{S}^{*}{ }_{2}$ & $\begin{array}{l}\text { Relative heat transfer area } \\
\text { of condensation section }\end{array}$ & 0.340 \\
\hline$T_{1}$ & $\begin{array}{l}\text { Operating temperature of } \\
\text { evaporation section }\end{array}$ & $90^{\circ} \mathrm{C}$ \\
\hline$T_{2}$ & $\begin{array}{l}\text { Working temperature of } \\
\text { condensation section }\end{array}$ & $15^{\circ} \mathrm{C}$ \\
\hline
\end{tabular}
saturated steam collide with the surface at equilibrium. According to the probability formula of collision between ideal gas and wall in statistical physics,

$$
\mathrm{k}=\frac{\mathrm{y}_{\mathrm{A}} \mathrm{PN}_{\mathrm{A}}}{2 \mathrm{R}} \sqrt{\frac{2 \mathrm{k}_{\mathrm{B}} \mathrm{T}}{\pi \mathrm{m}}} \mathrm{S}^{*}
$$

Table 2-2 The meanings and values of constants

The whole system is closed, participate in the reaction gas only include water vapor and other gases is not involved in the reaction, therefore, meet Raoult theorem (partial pressure on the part of the different components in the whole of a linear relationship) of the basic conditions, $\mathrm{p}_{\mathrm{A}}{ }^{*}$ : saturated vapor pressure, refers to the enclosed conditions of gas liquid equilibrium within the water vapor pressure of saturated condition. The value of saturated vapor pressure is related to the saturation temperature. When the temperature rises, the corresponding saturated vapor pressure rises. $\mathrm{P}_{\mathrm{B}}{ }^{*}$ : pressure of other gases in the current desalination plant environment.

The pressure of other gases in the environment,

$$
\left(\mathrm{p}_{\mathrm{A}}^{*}+\mathrm{p}_{\mathrm{B}}^{*}\right) \mathrm{V}=\mathrm{nRT}
$$

According to Raoult's theorem and other air insoluble in water, there is,

$$
\mathrm{P}=\mathrm{x}_{\mathrm{A}} \mathrm{p}_{\mathrm{A}}^{*} \mathrm{P}_{\mathrm{B}}=\mathrm{p}_{\mathrm{B}}^{*}
$$

According to the partial pressure ratio,

$$
\mathrm{P}=\mathrm{P}_{\mathrm{A}}+\mathrm{P}_{B}=x_{A} p_{A}^{*}+p_{B}^{*}, \mathrm{P}_{\mathrm{A}}=\mathrm{y}_{\mathrm{A}} \mathrm{p}
$$

The venturi tube reduces the vacuum in the working section to -81.2 , i. e. $20.1 \mathrm{kPa}$. At $90^{\circ} \mathrm{C}$, the saturated vapor pressure is $70.14 \mathrm{kPa}$. After evaporation, the concentration of the evaporated solution is $30 \%$, that is, the proportion of the solvent (water) is $70 \%$, the proportion of water vapor in the air, 


$$
\mathrm{y}_{\mathrm{A}}=\frac{\mathrm{x}_{\mathrm{A}} p_{A}^{*}}{\mathrm{P}_{\mathrm{A}}+\mathrm{P}_{\mathrm{B}}}=\frac{\mathrm{x}_{\mathrm{A}} p_{A}^{*}}{\mathrm{x}_{\mathrm{A}} p_{A}^{*}+\mathrm{p}_{\mathrm{B}}^{*}}=\frac{70 \% \times 70.14}{70 \% \times 70.14+20.1}=0.710
$$

By substituting the parameters in table 2-2, $\mathrm{y}_{\mathrm{A}}=0.710$ is obtained. The reaction rates of evaporation and condensation sections are as follows:

$$
\mathrm{k}_{1}=0.274, \mathrm{k}_{2}=0.116
$$

The reaction rate was substituted into the kinetic model, and the theoretical stable freshwater yield was 1 . 096L/10min.

\section{Benefit analysis of energy conservation and emission reduction}

(1) Small energy mainly comes from the solar desalination device, according to data shows the average earth orbit the sun radiation intensity of $1369 \mathrm{w} / \mathrm{m}^{2}$, on sea level for $1 \mathrm{kw} / \mathrm{m}^{2}$ standard peak intensity, in the islands of the south China sea near the equator region radiation intensity bigger, such as solar energy resources are very abundant, consumption has become increasingly scarce oil resources than the device adopts solar power supply energy to achieve the effective utilization of new energy.

(2) The daily output of the plant is 80 liters, equivalent to 160 bottles of mineral water, which can be supplied to about 31 adults for daily drinking, this will not only save the trouble of shipping fresh water affected by weather factors, but also save hundreds of tons of diesel every year, reduce a lot of carbon dioxide emissions, and achieve the effect of energy saving and emission reduction. This device can also solve the problem of fresh water drinking for the residents around the Salt Lake. Compared with the fresh water transported by vehicles that discharge a large amount of various pollution exhaust on the way, this device can achieve zero discharge of salt water desalination and no secondary pollution, save fuel resources and protect the ecological environment, and effectively reduce the emission of harmful gas.

(3) According to the mechanical structure of the device, the principle of loop heat pipe adopted in the device improves the heat transfer efficiency by about 4 times compared with the existing convective heat transfer; the designed condenser significantly increased the heat transfer contact surface area and increased the heat transfer efficiency by $15 \%$ compared with the traditional fin structure. The heat sink type turbulence evaporator is adopted, and the parallel arrangement greatly increases the heat transfer area and the heat transfer efficiency by $20 \%$.

\section{Acknowledgement}

National innovation and entrepreneurship training program for collegestudents20191049712013.

\section{References}

1. Zhang xiuzhi, wang jing, hao jian 'an et al. The utilization model of sea water resources in islands [J]. Water resources protection, 2015(3):115-118.

2. Xuan xiaomei et al. Comparison of seawater desalination technology and economic efficiency [J]. Steel \& iron, 2006, 41:634-637.

3. Li xuemin. Technical and economic analysis and comparison of major desalination methods [J]. First heavy technology, 2010, 3:63-70.

4. Xiao yashu et al. Development of integrated wind-wind-storage desalination device for island [J]. Industrial instrument and automation device, 2014, 2:105-108

5. Zhao yunmo. Study on low-temperature multi-effect distillation seawater desalination by solar energy [D]. Dalian university of technology, 2012.

6. Cao dejia. Research on the application of venturi pipe in flow control [J]. Value engineering, 2016, 35(19):145-146.

7. Qian jiyu. study on flow and heat transfer mechanism of capillary pump loop [D]. Nanjing university of science and technology, 2005. (in Chinese)

8. Chen xiaobo. Experimental study on two-phase capillary pump loop (CPL) [D]. Nanjing university of science and technology, 2004.

9. Wang xiaoli, zhang zunliang, tian Lin, huang pengfei, shan ke, wang shenghui. Market analysis and countermeasure research of small desalination plant $[\mathrm{J}]$. Salt science and chemical industry, 2008, 47(09):1-5. 